\title{
PRESIÓN DE GRUPO Y CONSUMO DE ALCOHOL EN ADOLESCENTES ESCOLARIZADOS
}

\author{
Juan Yovani Telumbre Terrero, Julieta Bolaños Reyes, Manuel Antonio López Cisneros, Lucely \\ Maas Góngora y Juan Pablo Sánchez Domínguez
}

Facultad de Ciencias de la Salud. Universidad Autónoma del Carmen. México

\begin{abstract}
Resumen
El consumo nocivo de alcohol provoca pérdidas sociales y económicas importantes, tanto para las personas como para la sociedad en su conjunto. El objetivo fue determinar la relación entre presión de grupo y consumo de alcohol en adolescentes escolarizados. Estudio transversal, descriptivo y correlacional, llevado a cabo en 298 adolescentes escolarizados de una institución pública de educación básica ciudad del Carmen, Campeche. Se utilizó el cuestionario de Resistencia a la Presión de Grupo en el Consumo de Alcohol en Adolescentes. Los datos fueron capturados y analizados en el programa estadístico SPSS Versión 23 a través de la estadística descriptiva e inferencial. Resultados: Se identificó una relación positiva y significativa de la presión de grupo general y el número de bebidas alcohólicas consumidas en un día típico, lo cual indica que a mayor presión grupal mayor consumo de alcohol ( $\mathrm{r}=.12 ; p=.044)$. El consumo de alcohol en los adolescentes va en incremento y constituyen un creciente problema de salud que pudiera ocasionar daños biológicos, psicológicos y sociales, a corto mediano y largo plazo.
\end{abstract}

Palabras clave: presión de grupo, consumo de alcohol, adolescentes.

\begin{abstract}
The harmful use of alcohol causes significant social and economic losses, both for individuals and for society. The objective was to determine the relationship between peer pressure and alcohol consumption in school adolescents. Cross-sectional, descriptive, and correlational study, carried out in 298 adolescents enrolled in a public institution of basic education Ciudad del Carmen, Campeche. The questionnaire on Resistance to Group Pressure in Alcohol Consumption in Adolescents was used. The data were captured and analyzed in the statistical program SPSS Version 23 through descriptive and inferential statistics. Results: A positive and significant relationship between general group pressure and the number of alcoholic beverages consumed on a typical day was identified, which indicates that the higher the group pressure, the higher the alcohol consumption $(\mathrm{r}=.12 ; \mathrm{p}=$ .044). Alcohol consumption in adolescents is increasing and constitutes a growing health problem that could cause biological, psychological, and social damage, in the short, medium, and long term.
\end{abstract}

Key words: peer pressure, alcohol consumption, adolescents.

\author{
Correspondencia \\ Juan Yovani Telumbre Terrero \\ Facultad de Ciencias de la Salud. Universidad Autónoma del Carmen. México \\ Email: jytt_@hotmail.com
}

Recibido: 2/04/2021

Aceptado: 4/05/2021 


\section{Introducción}

El consumo de alcohol provoca defunción y discapacidad a una edad relativamente temprana. En el grupo etario de 20 a 39 años, un $25 \%$ de las defunciones son atribuibles al consumo de alcohol. Existe una relación causal entre el consumo nocivo de alcohol y una serie de trastornos mentales y comportamentales, además de las enfermedades no transmisibles y los traumatismos. Más allá de las consecuencias sanitarias, el consumo nocivo de alcohol provoca pérdidas sociales y económicas importantes, tanto para las personas como para la sociedad en su conjunto (Organización Mundial de la Salud Alcohol [OMS], 2015).

Cada año se producen 3.3 millones de muertes en el mundo debido al consumo nocivo de alcohol, lo que representa un $5.9 \%$ de todas las defunciones. El uso nocivo de alcohol es un factor causal en más de 200 enfermedades y trastornos, en general, el $5.1 \%$ de la carga mundial de morbilidad y lesiones es atribuible al consumo de alcohol, calculado en términos de la esperanza de vida ajustada en función de la discapacidad (Organización de las Naciones Unidas [ONU], 2016). El consumo de alcohol es un problema de salud pública a nivel mundial, es un factor de riesgo para trastornos mentales y de la conducta, para hipertensión arterial sistémica, diabetes mellitus, cirrosis hepática y cáncer, entre otras. La OMS, menciona que en el 2014 el consumo de alcohol causa más de tres millones de muertes al año, representando el 5.9\% de todas las defunciones y el sexo masculino es el más afectado. Por otro lado, este organismo demuestra que el índice actual de consumo de alcohol aumenta según la edad, de menos de $1 \%$ a los 12 años a $19 \%$ a los 16 y 53\% a los 20 (OMS, 2015).

Aunque se han logrado avances, el consumo de alcohol entre menores continúa siendo un problema constante, cerca de 8.7 millones de estadounidenses entre los 12 y 20 años reportan que consumen alcohol en la actualidad. Esto representa casi $23 \%$ de este grupo de edad, para el cual el consumo de alcohol es ilegal. Para aquellos entre las edades de 12 y 20 años, el índice de consumo mensual reportado, incluido el consumo compulsivo de alcohol o en cantidades abundantes, disminuyó entre 2005 y 2014 (National Institute on Alcohol Abuse and Alcoholis , 2017).

En México, la Encuesta Nacional del Consumo de Drogas, Alcohol y Tabaco indica que la prevalencia del consumo de alcohol a nivel nacional corresponde al $51.4 \%$ para su población de 12 a 65 años; con respecto a la distribución por sexo, 62.7\% corresponde al masculino y 40.8\% al femenino11. En la población de 12 a 17 años se identificó una prevalencia del consumo de alcohol del 30\%, de los cuales 31.8\% corresponde al sexo masculino y $28.2 \%$ al sexo femenino. Situación que refleja la problemática de salud en los adolescentes, siendo esta etapa la más vulnerable en la cual

Know and Share Psychology, 2(2), 45-59 
se desarrollan hábitos de riesgo para su salud, entre los que destacan la presencia de alcoholismo, tabaquismo y farmacodependencia (Instituto Nacional de Psiquiatría Ramon de la Fuente Muñiz [INSRFM], Instituto Nacional de Salud Pública [INSR] y Secretaria de Salud [SSA], 2016).

Es cada vez más frecuente el consumo de alcohol en los jóvenes universitarios debido a la presión de grupo, lo cual constituye uno de los principales problemas a abordar, ya que a pesar de que el número de acciones de prevención adelantadas han ido en aumento en los últimos años si el problema persiste y se agrava. En décadas pasadas el problema del abuso en el consumo de alcohol era un asunto casi exclusivo del género masculino, en la actualidad ha trascendido las barreras de género y es día a día más frecuente que las mujeres beban y abusen de la bebida (Londoño, y Valencia, 2008; Perez, 2010).

Algunos jóvenes ceden ante la presión de grupo porque quieren caer bien, ser aceptados o porque temen que otras personas se burlen de ellos sino se unen al grupo. Otros ceden porque tienen curiosidad por probar algo nuevo que estos compañeros ya están haciendo. Muchas veces, un grupo de amigos adquiere gran importancia en la vida del adolescente y la presión que ejerce el grupo se convierte en un factor determinante. La presión de grupo es uno de los principales agentes socializadores, su influencia puede ser tanto positiva como negativa dependiendo del grupo al que se quiera pertenecer y la capacidad de rechazar las ideas, que, en la opinión de la persona, no son correctas (Del Toro et al, 2019).

La presión de grupo es uno de los medios que afecta a los adolescentes para iniciarse en el consumo de alcohol ya que no tienen la capacidad para abstenerse. Otro de los factores importantes para el consumo de alcohol es el entorno social, debido a que su ingesta en jóvenes es una conducta aceptada por la familia y la sociedad; incluso los adolescentes con padres consumidores son más propensos al abuso de dicha sustancia, en comparación a jóvenes con padres abstemios (Andrade et al, 2013). También se resalta que los adolescentes consumen alcohol como estrategia para afrontar los estresores propios de la vida académica y esto generalmente lo hacen en grupo con sus compañeros de estudio. De esta manera, un factor común es que la iniciación del consumo es con los pares estudiantes y la frecuencia de este se relaciona con el trato de los adolescentes con sus compañeros, por el deseo de ser aceptado en un grupo y las creencias positivas acerca de los efectos esperados de la bebida y para no ser rechazados por sus pares (Alvarado, Sánchez y Alvarado et al., López, 2014).

Know and Share Psychology, 2(2), 45-59 
El consumo de bebidas alcohólicas en las reuniones sociales es frecuente en muchos lugares del mundo, pero puede tener consecuencias sanitarias y sociales negativas relacionadas con sus propiedades tóxicas y la dependencia que puede producir. Además de las enfermedades crónicas que pueden contraer quienes beben grandes cantidades de alcohol a lo largo de varios años, el consumo de alcohol también se asocia a un aumento del riesgo de padecer afecciones agudas, tales como las lesiones, y en particular las provocadas por accidentes de tránsito (Orcasita, 2018).

Los datos expuestos en este trabajo sobre la presión de grupo, el uso y consumo de alcohol en adolescentes escolarizados y los nuevos patrones de consumo que se observan habitualmente en ellos, son un gran motivo de preocupación a todos los niveles. Es alarmante la repercusión y las consecuencias futuras de este consumo de riesgo en los más jóvenes, que se inician a edades cada vez más tempranas y que no suelen tener una conciencia real de la problemática implícita en ese consumo. El consumo que, por otra parte, no siempre se realiza como mera experimentación o conducta de rebeldía asociada a la edad.

Uno de los beneficios que se obtendrán de este proyecto es el de poder conocer la prevalencia tanto del consumo de alcohol como de las experiencias a las limitaciones del estudio en la población juvenil. La dimensión de los datos permitirá diseñar estrategias de prevención en estos jóvenes y conocer las asociaciones entre diferentes patrones de consumo y las variables que se asocian a estos modelos de consumo de alcohol así se logrará conocer más a fondo el porqué de ciertos patrones de consumo y se podrá incidir en la construcción de mejores planes de prevención del desarrollo de trastornos por abuso de esta sustancia y actuar en planes específicos de prevención y tratamiento así se podrían diseñar estrategias de cribaje de uso de alcohol en este grupo de jóvenes de alto riesgo, lo que podría disminuir la prevalencia de abuso de alcohol entre ellos.

\section{Objetivos}

1.- Describir la presión de grupo directa, indirecta y percibida por los adolescentes

2.- Estimar la prevalencia del consumo de alcohol en adolescentes

3.- Determinar la relación entre presión de grupo y consumo de alcohol en adolescentes escolarizados

Know and Share Psychology, 2(2), 45-59 


\section{Método}

Diseño del estudio: estudio de tipo transversal, descriptivo y correlacional.

Población, muestra y muestreo: la población de interés se conformó por 691 adolescentes escolarizados de una institución pública de educación básica ciudad del Carmen, Campeche. El tipo de muestreo fue aleatorio estratificado, considerando como estrato el sexo y el grado escolar de los participantes. El tamaño de la muestra será determinado en el paquete estadístico en n'Query Advisor Versión 4 para poblaciones finitas, con un nivel de significancia del 95\%, con un límite de error de estimación de .04 y un enfoque conservador del 50\%, por lo cual el tamaño de muestra fue de 298 .

Instrumento de medición: para el presente estudio se utilizó una Cedula de Datos Personales y Consumo de Alcohol, la cual estuvo constituida por dos secciones; la primera contiene tres preguntas abiertas sobre fecha de nacimiento, edad y grado escolar, dos preguntas dicotómicas para conocer el sexo y la ocupación del participante, así como dos preguntas de opción múltiple relacionadas con la ocupación y con quien vive el adolescente. La segunda sección abordó las prevalencias de consumo de alcohol, está sección está constituida por cuatro preguntas dicotómicas, además de dos preguntas abiertas vinculadas con edad de inicio y numero de bebidas alcohólicas que consumen en un día típico. Se abordará el consumo de alcohol, alguna vez en la vida, en el último año, en los últimos treinta días y en los últimos siete días.

Además, se utilizó el cuestionario de Resistencia a la Presión de Grupo en el Consumo de Alcohol en Adolescentes (CRPG) elaborado por Londoño et al (2007), está compuesto por 45 reactivos que describen situaciones que pueden representar presión al adolescente, las opciones de respuesta están dadas en escala tipo Likert del 1 al 4 para evaluar la percepción de presión del sujeto o que tan presionado se siente el adolescente ante situaciones en las que el grupo interviene. El cuestionario consta de dos subescalas, la primera denominada: resistencia a la presión de grupo directa que incluye los reactivos 1 al 18; la subescala 2 llamada: resistencia a la presión de grupo indirecta que incluye los reactivos 19 al 40. Además, se incluye una tercera subescala que mide el nivel de presión percibida antes situaciones específicas evaluadas a través de los reactivos 41 al 45 . El cuestionario incluye además cuatro preguntas sobre el consumo de alcohol, tipo de bebida ingerida y la última ocasión en la que se embriago.

Know and Share Psychology, 2(2), 45-59 
La calificación de cada subescala se obtiene al dar una puntuación de 1 al 4 según sea la situación, puntajes altos indican mayor habilidad para resistir la presión de grupo y los puntajes bajos menor habilidad y, por tanto, mayor riesgo de consumo abusivo. La validación del instrumento se hizo con la evaluación de dos jurados expertos y la aplicación de la prueba de confiabilidad reportando un Alpha de Cronbach de 0.91 (Londoño et al, 2007), en este estudio se obtuvo una confiabilidad de .94.

Procedimiento para la recolección de datos: se solicitó la evaluación del estudio por parte del Comité de Investigación de la Facultad de Ciencias de la Salud de la Universidad Autónoma del Carmen. Posteriormente se solicitó la autorización por escrito de la institución educativa donde se realizó el estudio, una vez obtenida la autorización se solicitó el listado de alumnos para realizar la selección de manera aleatoria y estratificada de acuerdo con el sexo y grado escolar. Identificados los participantes, se contactaron en el horario y día acordado por la institución y se les realizó la invitación de manera verbal para participar en el estudio.

Aquellos adolescentes menores de edad que aceptaron participar, se les hizo entrega del consentimiento informado para padres o tutores. Aquellos adolescentes que se les autorizo participar se les hizo entrega de un asentimiento informado, si se trató de participantes mayores de edad se hizo entrega del consentimiento informado donde se explicó de manera clara, precisa y concisa los propósitos de la investigación. Posterior a la firma del asentimiento o consentimiento informado se les hizo entrega de los instrumentos de medición, el cual se conformó bajo el siguiente orden: Cedula de Datos Personales y la Escala de Resistencia a la Presión de Grupo en el Consumo de Alcohol. Se recalcó en todo momento que la participación fue totalmente voluntaria y confidencial y que la información proporcionada no repercutirá en su formación académica. Culminado el llenado de los instrumentos se agradeció la participación de los sujetos.

Consideraciones éticas: el estudio se apegó al Reglamento de la Ley General de Salud en Materia de Investigación para la Salud (SSA, 1987).

Análisis de resultados: los datos fueron capturados y analizados en el programa estadístico SPSS Versión 23, se determinó la confiabilidad de los instrumentos a través del Alpha de Cronbach, así también se utilizó la estadística descriptiva por medio de frecuencias, proporciones y medidas de tendencia central y de dispersión. Se determinó la normalidad de las variables continuas por medio de la Prueba de Kolmogorov-

Know and Share Psychology, 2(2), 45-59 
Smirnov y se decidió en la utilización de pruebas paramétricas a través de la Correlación de Pearson.

\section{Resultados}

\section{Características sociodemográficas}

E1 50.3\% de la muestra corresponde al sexo femenino y el $92.5 \%$ reportan un estado civil soltero. En cuanto al grado escolar el $45.9 \%$ cursa el segundo de grado de secundaria y que el $19.0 \%$ de los estudiantes trabaja, de estos, el $94.6 \%$ se enfoca a actividades no profesionistas. El 62.6\% refirió vivir actualmente con ambos padres.

\section{Consumo de alcohol}

La media de edad de los participantes fue de 13.01 años (DE: 1.23), con una edad de inicio del consumo de alcohol de 11.00 (DE:1.48). Los adolescentes consumen en promedio 2.19 (DE: 1.09) bebidas alcohólicas en un día típico, con un minino de 1 hasta 5 bebidas. Con relación a la prevalencia del consumo de alcohol se pudo constatar que del total de los participantes el $38.4 \%$ ha consumido alcohol alguna vez en la vida, el $24.5 \%$ en el último año, $22.1 \%$ en el último mes y $21.4 \%$ en la última semana (Tabla 1 ).

\section{Tabla 1}

Prevalencias del consumo de alcohol.

\begin{tabular}{lllll}
\hline \multirow{2}{*}{ Prevalencia } & Si & \multicolumn{3}{c}{ No } \\
\cline { 2 - 5 } & $f$ & $\%$ & $f$ & $\%$ \\
\hline Alguna vez en la vida & 113 & 38.4 & 181 & 61.6 \\
En el último año & 72 & 24.5 & 222 & 75.5 \\
En el último mes & 65 & 22.1 & 229 & 77.9 \\
En la última semana & 63 & 21.4 & 231 & 78.6 \\
\hline
\end{tabular}

Nota: $f=$ frecuencia, $\%=$ Porcentaje.

\section{Resistencia a la presión de grupo directa}

Por lo que respecta a la resistencia a la presión de grupo directa en los estudiantes de secundaria, se pudo identificar que el $75.2 \%$ está totalmente en desacuerdo al verse obligado a tomar porque le es difícil decir no, el 74.5\% terminan tomando si ellos los excluyen del grupo y el $74.1 \%$ piensa que está obligado a tomar si su acompañante 
toma. Mientras que $48.3 \%$ está totalmente de acuerdo en controlar este tipo de situación no consumiendo, el $42.9 \%$ piensan que sus amigos los comprenden y el $38.4 \%$ cuenta con la sufriente autonomía para no dejarse presionar (Tabla 2).

\section{Tabla 2}

Frecuencia y proporciones de la Resistencia a la presión de grupo directa.

\begin{tabular}{|c|c|c|c|c|c|c|c|c|}
\hline \multirow[t]{2}{*}{ Ítem } & \multicolumn{2}{|c|}{$\begin{array}{l}\text { Totalmente en } \\
\text { desacuerdo }\end{array}$} & \multicolumn{2}{|c|}{ En desacuerdo } & \multicolumn{2}{|c|}{ De acuerdo } & \multicolumn{2}{|c|}{$\begin{array}{l}\text { Totalmente de } \\
\text { acuerdo }\end{array}$} \\
\hline & $f$ & $\%$ & $f$ & $\%$ & $f$ & $\%$ & $f$ & $\%$ \\
\hline \multicolumn{9}{|c|}{ Cuando te encuentras en una reunión en las que ofrecen bebidas alcohólicas } \\
\hline $\begin{array}{l}\text { Te ves obligado a tomar porque te } \\
\text { es difícil decir no }\end{array}$ & 221 & 75.2 & 45 & 15.3 & 15 & 5.1 & 13 & 4.4 \\
\hline $\begin{array}{l}2 . \quad \text { Intentas mantener alejado de la } \\
\text { persona encargada de servir los tragos }\end{array}$ & 96 & 32.7 & 47 & 16.0 & 64 & 21.8 & 87 & 29.6 \\
\hline $\begin{array}{l}\text { 3. Controlas la cantidad de alcohol } \\
\text { que consumes }\end{array}$ & 101 & 34.4 & 27 & 9.2 & 40 & 13.6 & 126 & 42.9 \\
\hline $\begin{array}{l}\text { 4. Recibes el trago que te ofrecen y } \\
\text { finges que lo tomas }\end{array}$ & 137 & 46.6 & 49 & 16.1 & 40 & 13.6 & 68 & 23.1 \\
\hline $\begin{array}{l}\text { 5. Controlas este tipo de situación } \\
\text { no consumiendo }\end{array}$ & 93 & 31.6 & 22 & 7.5 & 37 & 12.6 & 142 & 48.3 \\
\hline \multicolumn{9}{|c|}{ Cuando te encuentras con tus amigos y NO deseas tomar: } \\
\hline $\begin{array}{l}\text { 6. Si todos están tomando piensas } \\
\text { que estás obligado a tomar }\end{array}$ & 210 & 71.4 & 59 & 20.1 & 9 & 3.1 & 15 & 5.1 \\
\hline $\begin{array}{l}\text { 7. Si se burlan cuando no tomas } \\
\text { piensas que estás obligado a tomar }\end{array}$ & 208 & 70.7 & 51 & 17.3 & 17 & 5.8 & 18 & 6.1 \\
\hline $\begin{array}{l}\text { 8. Cuentas con la suficiente } \\
\text { autonomía para no dejarte presionar }\end{array}$ & 92 & 13.1 & 40 & 13.6 & 49 & 16.7 & 113 & 38.4 \\
\hline $\begin{array}{l}\text { 9. Piensas que tus amigos son firmes } \\
\text { en la decisión de no tomar }\end{array}$ & 97 & 33.0 & 44 & 15.0 & 66 & 22.4 & 87 & 29.6 \\
\hline $\begin{array}{l}10 . \quad \text { Terminas tomando si la ocasión } \\
\text { es especial }\end{array}$ & 178 & 60.5 & 45 & 15.3 & 35 & 11.9 & 36 & 12.2 \\
\hline $\begin{array}{l}\text { 11. Piensas que tus amigos te } \\
\text { comprenden }\end{array}$ & 87 & 29.6 & 29 & 9.9 & 52 & 17.7 & 126 & 42.9 \\
\hline $\begin{array}{l}\text { 12. Terminas tomando si ellos ejercen } \\
\text { presión }\end{array}$ & 212 & 72.1 & 54 & 18.4 & 16 & 5.4 & 12 & 4.1 \\
\hline $\begin{array}{l}\text { 13. Terminas tomando si ellos te } \\
\text { excluyen del grupo }\end{array}$ & 219 & 74.5 & 52 & 17.7 & 12 & 4.1 & 11 & 3.7 \\
\hline $\begin{array}{l}\text { 14. Tiendes a tomar más alcohol } \\
\text { cuando estas sin compañía }\end{array}$ & 216 & 73.5 & 50 & 17.0 & 13 & 4.4 & 15 & 5.1 \\
\hline $\begin{array}{l}\text { 15. Terminas tomando si percibes si } \\
\text { no te creen las excusas que diste }\end{array}$ & 208 & 70.7 & 53 & 18.0 & 15 & 5.1 & 18 & 6.1 \\
\hline $\begin{array}{l}\text { 16. Terminas tomando si la persona } \\
\text { que te gusta te ofrece alcohol }\end{array}$ & 208 & 70.7 & 54 & 18.4 & 15 & 5.1 & 17 & 5.8 \\
\hline $\begin{array}{l}\text { 17. Si a alguien le gusta tomar } \\
\text { piensas que estás obligado }\end{array}$ & 191 & 65.0 & 67 & 22.8 & 19 & 6.5 & 17 & 5.8 \\
\hline $\begin{array}{l}\text { 18. Si tu acompañante toma piensas } \\
\text { que estás obligado a tomar }\end{array}$ & 218 & 74.1 & 56 & 19.0 & 9 & 3.1 & 11 & 3.7 \\
\hline
\end{tabular}

Nota: $f=$ frecuencia, $\%=$ Porcentaje.

Know and Share Psychology, 2(2), 45-59 


\section{Resistencia a la presión de grupo directa}

Se pudo observar que el $88.8 \%$ está totalmente en desacuerdo en sentirse excluido y termina tomando, el $87.1 \%$ ridiculizado y termina tomando y el $86.4 \%$ sentirse vulnerable y terminar tomando. Así como el 50.7\% está totalmente de acuerdo en no desear tomar y sentirse firme en su decisión, el $40.1 \%$ en querer tomar una bebida sin alcohol a comer otra cosa y el $26.9 \%$ en sentirse con la libertad de no tomar

\section{Tabla 3}

Frecuencia y proporciones de la Resistencia a la presión de grupo indirecta.

\begin{tabular}{|c|c|c|c|c|c|c|c|c|c|}
\hline & & \multicolumn{2}{|c|}{$\begin{array}{l}\text { Totalmente en } \\
\text { desacuerdo }\end{array}$} & \multicolumn{2}{|c|}{ En desacuerdo } & \multicolumn{2}{|c|}{ De acuerdo } & \multicolumn{2}{|c|}{$\begin{array}{l}\text { Totalmente de } \\
\text { acuerdo }\end{array}$} \\
\hline & & $f$ & $\%$ & $f$ & $\%$ & $f$ & $\%$ & $f$ & $\%$ \\
\hline \multicolumn{10}{|c|}{ Si una persona del grupo que tu admiras toma y tú NO, piensas que: } \\
\hline 19. & Es más deseable que tú & 163 & 55.4 & 86 & 29.3 & 16 & 5.4 & 29 & 9.9 \\
\hline 20. & Es más reconocido que tú & 150 & 51.0 & 77 & 26.2 & 43 & 14.6 & 24 & 8.2 \\
\hline 21. & Es más maduro que tú & 168 & 57.1 & 79 & 26.9 & 22 & 7.5 & 25 & 8.5 \\
\hline 22. & Es más controlada que tú & 180 & 61.2 & 63 & 21.4 & 32 & 10.9 & 19 & 6.5 \\
\hline 23. & Es más libre que tú & 136 & 46.3 & 68 & 23.1 & 56 & 19.0 & 34 & 11.6 \\
\hline 24. & Es más sociable que tú & 146 & 49.7 & 71 & 24.1 & 42 & 14.3 & 35 & 11.9 \\
\hline 25. & Es más moderno/a que tú & 152 & 51.7 & 76 & 25.9 & 38 & 12.9 & 28 & 9.5 \\
\hline $\begin{array}{l}26 . \\
\text { grup }\end{array}$ & $\begin{array}{l}\text { Es más, tenido en cuenta por el } \\
\text { ue tú }\end{array}$ & 148 & 50.3 & 81 & 27.6 & 31 & 10.5 & 34 & 11.6 \\
\hline 27. & Es más agradable que tú & 165 & 56.1 & 82 & 27.9 & 19 & 6.5 & 28 & 9.5 \\
\hline \multicolumn{10}{|c|}{ Cuando el grupo no está de acuerdo con tu decisión de NO tomar te sientes: } \\
\hline 28. & Incómodo y terminas tomando & 237 & 80.6 & 40 & 13.6 & 9 & 3.1 & 8 & 2.7 \\
\hline 29. & Rechazado y terminas tomando & 252 & 85.7 & 26 & 8.8 & 11 & 3.7 & 5 & 1.7 \\
\hline 30. & Deprimido y terminas tomando & 247 & 84.0 & 26 & 8.8 & 8 & 2.7 & 13 & 4.4 \\
\hline 31. & Con la libertad de no tomar & 167 & 56.8 & 27 & 9.2 & 21 & 7.1 & 79 & 26.9 \\
\hline 32. & Vulnerable y terminas tomando & 254 & 86.4 & 28 & 9.5 & 4 & 1.4 & 8 & 2.7 \\
\hline 33. & Excluido y terminas tomando & 261 & 88.8 & 22 & 7.5 & 5 & 1.7 & 6 & 2.0 \\
\hline 34. & Ridiculizado y terminas tomando & 256 & 87.1 & 25 & 8.5 & 8 & 2.7 & 5 & 1.7 \\
\hline 35. & Aburrido y terminas tomando & 249 & 84.7 & 27 & 9.2 & 8 & 2.7 & 10 & 3.4 \\
\hline \multicolumn{10}{|c|}{ Estas en una reunión en la que ofrecen bebidas alcohólicas y NO deseas tomar, tú dices que: } \\
\hline 36. & Estas enfermo y no puedes toma & 197 & 67.0 & 31 & 10.5 & 22 & 7.5 & 44 & 15.0 \\
\hline $\begin{array}{l}37 . \\
\text { no p }\end{array}$ & $\begin{array}{l}\text { Estas tomando medicamento y } \\
\text { es tomar }\end{array}$ & 199 & 67.7 & 28 & 9.5 & 23 & 7.8 & 4 & 15.0 \\
\hline $\begin{array}{l}38 . \\
\text { toma }\end{array}$ & Te tienes que ir y no puedes & 191 & 65.0 & 25 & 8.5 & 23 & 7.8 & 55 & 18.7 \\
\hline $\begin{array}{l}39 . \\
\text { alcol }\end{array}$ & $\begin{array}{l}\text { Quieres tomar una bebida sin } \\
\text { a comer otra cosa }\end{array}$ & 128 & 43.5 & 20 & 6.8 & 28 & 9.5 & 118 & 40.1 \\
\hline $\begin{array}{l}40 . \\
\text { tu de }\end{array}$ & $\begin{array}{l}\text { No deseas tomar y eres firme en } \\
\text { ión }\end{array}$ & 110 & 37.4 & 13 & 4.4 & 22 & 7.5 & 149 & 50.7 \\
\hline
\end{tabular}

Nota: $f=$ frecuencia, $\%=$ Porcentaje.

Know and Share Psychology, 2(2), 45-59 
Con relación a la presión percibida, los hallazgos muestran que el $83.3 \%$ está totalmente en desacuerdo cuando sus amigos se burlan porque no desean tomar, el $76.9 \%$ cuando sus amigos los excluyen de sus actividades por no querer tomar y el $71.1 \%$ cuando están con una persona que les gusta. Mientras que el 11.2\% está totalmente de acuerdo cuando celebran una fecha especial y no los invitan, el 7.8\% cuando están con una persona que les gusta y el 6.5\% cuando sus amigos los excluyen de sus actividades por no querer tomar.

\section{Tabla 4}

Frecuencia y proporciones de los adolescentes sobre la presión percibida.

\begin{tabular}{|c|c|c|c|c|c|c|c|c|c|}
\hline \multirow[t]{2}{*}{ Ítems } & & \multicolumn{2}{|c|}{$\begin{array}{l}\text { Totalmente en } \\
\text { desacuerdo }\end{array}$} & \multicolumn{2}{|c|}{$\begin{array}{l}\text { En } \\
\text { desacuerdo }\end{array}$} & \multicolumn{2}{|c|}{ De acuerdo } & \multicolumn{2}{|c|}{$\begin{array}{l}\text { Totalmente } \\
\text { de acuerdo }\end{array}$} \\
\hline & & $f$ & $\%$ & $f$ & $\%$ & $f$ & $\%$ & $f$ & $\%$ \\
\hline & $\begin{array}{l}41 . \quad \text { ¿Cuándo tus } \\
\text { amigos se burlan porque } \\
\text { no deseas tomar? }\end{array}$ & 245 & 83.3 & 27 & 9.2 & 9 & 3.1 & 13 & 4.4 \\
\hline & $\begin{array}{l}42 . \quad \text { ¿Cuándo tus } \\
\text { amigos insisten para que } \\
\text { accedas a tomar al } \\
\text { menos un trago? }\end{array}$ & 202 & 68.7 & 55 & 18.7 & 22 & 7.5 & 15 & 5.1 \\
\hline & $\begin{array}{l}43 . \quad ¿ \text { Cuándo estas } \\
\text { con una persona que te } \\
\text { gusta? }\end{array}$ & 209 & 71.1 & 40 & 13.6 & 22 & 7.5 & 23 & 7.8 \\
\hline & $\begin{array}{l}44 . \quad \text { ¿Cuándo tus } \\
\text { amigos te han excluido } \\
\text { de tus actividades por } \\
\text { no querer tomar? }\end{array}$ & 226 & 76.9 & 28 & 9.5 & 22 & 7.5 & 18 & 6.5 \\
\hline & $\begin{array}{l}45 . \quad \text { ¿Cuándo } \\
\text { celebran una fecha } \\
\text { especial y no te invitan? }\end{array}$ & 193 & 65.6 & 50 & 17.0 & 18 & 6.1 & 33 & 11.2 \\
\hline
\end{tabular}

Para dar respuesta al objetivo general del estudio, se realizó una prueba de correlación, donde se identificó una relación positiva y significativa de la presión de grupo general y el número de bebidas alcohólicas consumidas en un día típico, lo cual indica que a mayor presión grupal mayor consumo de alcohol $(\mathrm{r}=.12 ; p=.044)$.

\section{Discusión}

En cuanto a las características sociodemográficas se identificó que el 19.0\% trabaja, de los cuales el $94.6 \%$ son no profesionistas, estos datos son similares a lo reportado por Medina et al (2017). quien señala que el 13.2\% \% de los adolescentes 
estudian y trabajan simultáneamente. Esto pudiera deberse a que el trabajo les permite solventar sus gastos personales, ayudar en los gastos del hogar, continuar con sus estudios y principalmente volverse dependientes de sus padres. Del Toro et al (2019) puntualizan que la combinación de estudiar y trabajar de los adolescentes es una estrategia para asegurar la reproducción material de las familias en casos de escasez de recursos económicos. Por lo tanto, la combinación de las actividades escolares y laborales puede entenderse como una respuesta de los jóvenes ante las estrategias de sobrevivencia de los grupos domésticos para hacer frente a las dificultades económicas.

Se identificó que el $62.6 \%$ de los adolescentes viven con ambos padres, vivir con ambos padres permite que los adolescentes se desarrollen de una manera eficaz, con todas las habilidades que les permitan desarrollarse en la vida adulta. Orcasita (2018) analizaron los recursos psicosociales de autoestima y apoyo social percibido como variables mediadoras en la relación entre la calidad de la comunicación familiar y el ánimo depresivo. Por otro lado, se destaca la importancia de la familia para el desarrollo de la autoestima infantil, reconociendo la importancia de la función de los padres.

Los participantes iniciaron el consumo de alcohol en promedio a los 11 años, lo cual es menor a lo reportado con Mayorga-Lascano et al (2019), quienes mencionan que la edad promedio del consumo de alcohol por primera vez fue a los 15.8 años, siendo la edad mínima a los 9 años y la máxima a los 24 años. Situación que refleja la problemática de salud en los adolescentes, siendo esta etapa la más vulnerable en la cual se desarrollan hábitos de riesgo para su salud, entre los que destacan la presencia de alcoholismo.

Las prevalencias del consumo de alcohol fueron menores con relación a los reportado por Romero et al (2019). Esto pudiera deberse a que la adolescencia es el periodo en el que probablemente aparece el hábito social de consumo de alcohol, es un periodo de transición entre la infancia y la adultez, en el cual el adolescente se siente miembro y partícipe de una "cultura de edad" caracterizada por sus propios comportamientos, valores, normas, espacios y modas y los valores que por la interacción grupal que interioriza van a contribuir a la construcción de su identidad personal (Pérez, 2010; Navarro et al, 2020).

Por lo que respecta a la resistencia a la presión de grupo directa se identificó que el $75.2 \%$ está totalmente en desacuerdo al verse obligado a tomar porque le es difícil decir no y el $74.5 \%$ está totalmente en desacuerdo en que terminan tomando si ellos los excluyen del grupo, esto es similar a los resultados de Campo-Arias et al (2013) quienes reportaron que los participantes presentan altos niveles de habilidad para resistirse a la presión de grupo directa. Lodoño y Valencia (2008) señalan que la importancia que

Know and Share Psychology, 2(2), 45-59 
atribuyen los jóvenes a ser aceptados y reconocidos por otros, como parte de la búsqueda de identidad en la etapa de la adolescencia, contribuye a que los chicos terminen cediendo a la presión de grupo; propiciando así el consumo de alcohol.

Con respecto a la presión de grupo indirecta se observa que el $88.8 \%$ está totalmente en desacuerdo en sentirse excluido y terminar tomando y el $87.1 \%$ está totalmente en desacuerdo en sentirse ridiculizado, lo que es menor en comparación con los hallazgos de Alvarado et al (2014) Por lo que se considera que las personas con dicha resistencia dan a conocer de forma espontánea sus gustos e intereses, pueden aceptar comentarios sin sentirse incómodos, diferir con las personas explícitamente, y responder con un "no" ante las situaciones en las que no esté de acuerdo (Vera, et al., 2012).

Respecto a la presión de grupo percibida se muestra que el $83.3 \%$ está totalmente en desacuerdo cuando sus amigos se burlan porque no desean tomar, el 76.9\% está totalmente en desacuerdo cuando sus amigos los excluyen de sus actividades por no querer tomar y el $71.1 \%$ está totalmente en desacuerdo cuando están con una persona que les gusta, similar a los estudios de Amiegheme (2012), de esta manera se menciona que la presión de las amistades puede suscitar en el adolescente sensaciones de rechazo, búsqueda de aprobación o, por el contrario, puede ocurrir que el adolescente no permita que los comentarios y comportamientos que realizan los pares influyan sobre él, lo cual depende de que tan presionado se sienta ante la situación. Sin embargo, los adolescentes que tienen niveles bajos de resistencia a la presión de grupo presentan niveles altos de consumo de alcohol (Acosta et al, 2011).

En cuanto a la relación positiva y significativa de la presión de grupo general y el número de bebidas alcohólicas consumidas en un día típico, indica que a mayor presión grupal mayor consumo de alcohol lo cual concuerda con Zavala y Vásquez (2016). Estos autores señalan que la asertividad y habilidades sociales juegan un papel muy importante para resistirse a la presión que ejercen las amistades, lo cual hace suponer que estos alumnos consumen alcohol por la influencia de los pares y no por decisión propia. Esto implica reforzar que el asertividad, autoestima, inteligencia emocional, entre otros, que permitan a los jóvenes realizar un juicio crítico frente a las situaciones del consumo. El consumo frecuente de alcohol es una problemática multifactorial donde además de la presión del grupo de pares influyen factores ambientales, culturales, personales y biológicos que inciden en el consumo de la sustancia (Andrade et al, 2013).

Know and Share Psychology, 2(2), 45-59 


\section{Conclusión}

El análisis de la información permitió corroborar que el consumo de alcohol en los adolescentes va en incremento y constituyendo un creciente problema de salud que pudiera ocasionar daños biológicos, psicológicos y sociales, a corto mediano y largo plazo. En la adolescencia se conjugan una serie de factores que retardan o limitan dicha conducta; destacándose la importancia de la familia como eje principal en la adquisición de conductas saludables o no saludables. Así también el papel de los aspectos sociales contribuye al inicio o mantenimiento de la ingesta de bebidas alcohólicas y, en su caso, desarrollar un trastorno de dependencia están determinados en función de la cantidad y frecuencias de la ingesta de bebidas alcohólicas. Por lo tanto, es necesario fortalecer acciones específicas, desde el ámbito familiar y social, que permitan brindar las habilidades sociales necesarias para resistir a la presión de pares con relación al consumo de alcohol, tabaco y otras drogas.

\section{Referencias}

Acosta, L. D., Fernández, A. R. y Pillon, S. C. (2011). Factores sociales para el uso de alcohol en adolescentes y jóvenes. Revista Latino-Americana de Enfermagem. 19(SPE); 771-781. https://doi.org/10.1590/S010411692011000700015

Alvarado, M. C. R., Sánchez, C. M. P. y López, J. D. M. (2014). Resistencia de la presión de grupo y consumo de alcohol en adolescentes. Revista de Psicología Universidad de Antioquia. 6(1); 25-40.

\section{https://revistas.udea.edu.co/index.php/psicologia/article/view/21615}

Amiegheme, F. E. (2013). Psychosocial factors affecting adolescent alcohol abuse in Edo state, Nigeria. Archives of Applied Science Research. 5(1); 88-92.

Andrade, S. J. A., Yepes, T. Á. y Sabogal, G. M. L. (2013). Resistencia a la presión de grupo y consumo de alcohol en 50 jóvenes universitarios entre los 16 y 22 años de la Universidad de San Buenaventura Medellín extensión Ibagué. Revista Colombiana de Ciencias Sociales. 4(1); 11-22. https://www.redalyc.org/articulo.oa?id=497856284003

Campo-Arias, A., Villamil-Vargas, M. y Herazo, E. (2013). Confiabilidad y dimensionalidad del AUDIT en estudiantes de medicina. Psicología desde el Caribe. 30(1); 21-35. 
http://www.scielo.org.co/scielo.php?script=sci_arttextypid=S0123417X2013000100003ylng=enytlng=.

Del Toro Kondeff, M., Caraballosa, D. L. y Castro, M. S. (2019). Patrones de consumo de alcohol en adolescentes. Revista del Hospital Psiquiátrico de La Habana. 15(2); 1-7. http://www.revhph.sld.cu/index.php/hph/article/view/52

Elashoff, D.J., Dixon, J.W., Crede, M. K. y Fothenringham, (2000). N. n.QueryAdvisior (version 4.0) Copyrigth (software estadístico para PC). Los Ángeles, CA: Statistical Solutions.

Instituto Nacional de Psiquiatría Ramón de la Fuente Muñiz; Instituto Nacional de Salud Pública; Comisión Nacional Contra las Adicciones, y Secretaría de Salud. Encuesta Nacional de Consumo de Drogas, Alcohol y Tabaco 2016-2017: Reporte de Alcohol. México; 2017. https://doi.org/10.17711/sm.0185-3325.2018.003

Londoño, P. C. y Valencia, L.C. (2008). Asertividad, resistencia a la presión de grupo y consumo de alcohol en universitarios. Acta Colombiana de Psicología.11 (1); 155-165. http://hdl.handle.net/10983/599

Londoño, P. C., Valencia, L. S. C., Sánchez, L. y León, V. (2007). Diseño del cuestionario resistencia a la presión de grupo en el consumo de alcohol (CRPG). Suma Psicológica. 14 (2);271-288.

Mayorga-Lascano, M., Moreta-Herrera, R., León-Tamayo, L. y Troncozo-Guevara, B. (2019). Actitudes favorables y consumo de alcohol en adolescentes ecuatorianos. Health y Addictions/Salud y Drogas.19(2); 1-13.

Medina, M. E. G., Facundo, F. R. G., Cortez, J. G. A., Castillo, M. M. A. y da Silva, E. C. (2017). Autoestima y consumo de alcohol en adolescentes escolarizados. NURE investigación: Revista Científica de enfermería. 14 (88); 1 7.

National Institute on Alcohol Abuse and Alcoholis. (2017). Strategic Plan 2017-2021. Disponible en: https://www.niaaa.nih.gov/sites/default/files/ StrategicPlan_NIAAA_optimized_2017-2020.pd

Navarro, O.E.I.P., Alonso, C.M.M., Guzmán, F.F.R., Álvarez, B.J. y Rodríguez, P.L.A. (2020). Use of social media and mass interpersonal persuasion: predictors of alcohol consumption in young people. SMAD, Rev Eletrônica Saúde Mental Álcool Drog. 16(1); 1-8.

Orcasita, L. T. (2018). Factores psicosociales asociados a los patrones de consumo de alcohol en adolescentes escolarizados. Psicología desde el Caribe. 35(1); 33-48. 
Organización de las Naciones Unidas [ONU] (2016). Urbanization and Development: Emerging Futures. Recuperado de: http://cdn.plataformaurbana.cl/wpcontent/uploads/2016/06/wcrfull-report-2016.pdf

Organización Mundial de la Salud, OMS (2015). Informe de situación regional sobre el alcohol y la salud en las Américas. Washington, DC: OPS.

https://www.paho.org/hq/dmdocuments/2015/alcohol-informe-salud-americas2015.pdf

Perez, C. L. (2010). Resistencia de la presión de grupo, creencias acerca del consumo y consumo de alcohol en universitarios. Anales de Psicología/Annals of Psychology. 26 (1); 27-33. https://revistas.um.es/analesps/article/view/91931

Romero, M. D. L. R., Oyervide, J. A. J. y Malla, M. I. M. (2019). Determinantes sociales del consumo de alcohol en adolescentes del colegio Daniel Córdova de Cuenca: Estudio transversal, 2016. Revista de la Facultad de Ciencias Médicas de la Universidad de Cuenca. 37(3); 54-61.

Secretaría de Salud (1987). Reglamento de la Ley general de salud en materia de investigación para la salud. Disponible en: Recuperado de http://www.salud.gob.mx/unidades/cdi/nom/ compi/rlgsmis.html

Vera, N. C. D., Aguilera, M., Batura, R., Campos, F., Canese, M., González, A., ...y Triderman, P. (2012). Presión de grupo y consumo de alcohol en estudiantes del curso probatorio de ingreso de universidad privada. Eureka (Asunción) en Línea. 9(2); 196-216. http://pepsic.bvsalud.org/scielo.php?script=sci_arttextypid=S222090262012000200007 ylng=ptytlng=es.

Zavala, R. L. y Vasquez, L. (2016). Influencia de los estilos de crianza y la resistencia a la presión de grupo sobre el consumo de alcohol en adolescentes de la ciudad de Cajamarca. Revista Perspectiva. 17(1); 33-45

Know and Share Psychology, 2(2), 45-59 